\title{
Seeing Data in a New Light: Data Visualization Techniques to Improve Understanding
}

\author{
Shannon DeWitt \\ Centers for Disease Control and Prevention, Atlanta, Georgia, United States \\ Objective
}

The presenter will demonstrate complex health and environment surveillance data visualization techniques within the CDC's Environmental Public Health Tracking Network.

\section{Introduction}

In 2002, the United States (US) Centers for Disease Control and Prevention (CDC) launched the National Environmental Public Health Tracking Program (Tracking Program) to address the challenges and gaps in the nation's environmental health surveillance infrastructure. The Tracking Program's mission is to provide information from a nationwide network of integrated health and environmental data that drives actions to improve the health of communities. As a primary objective of the Tracking Program, the Environmental Public Health Tracking Network (Tracking Network) was developed as an online surveillance system with data available for 23 topics and over 450 different health, environmental, and population measures. The integration and display of such disparate data can be challenging. For data consumers without scientific training, or even scientists and public health professionals with limited time, it can be difficult to examine and explore the data in an online surveillance system. Additionally, casual data consumers may not require complex data details; a big picture perspective may be appropriate to their needs. The Tracking Network - which applies standardized data, a modern user interface, techniques catering to a variety of data consumers, and best practices in data visualization - provides a dynamic data query system that allows users to visualize different types of environmental health data in numerous ways including a variety of charting, mapping, and graphing options.

\section{Methods}

After the Tracking Program identifies important environmental health topics, data standards are developed to aid in data validation and to ensure consistency and comparability of the data. The data are aggregated into standardized stratifications, summarized, and used to calculate environmental health measures. Visualization requirements for each measure are determined and developed on the Tracking Network. In addition, public health content are developed to provide important context for the user. The final step is the release of the environmental health measures onto the National Tracking Network, where they can be queried, visualized, compared, and analyzed with all of the other environmental health measures on the Tracking Network.

\section{Results}

Launched in 2009, the Tracking Network developed at CDC is home to over 450 standardized environmental health measures spanning 23 topics and multiple years, and can be displayed at a geographic level of state, county, and census tract. The Tracking Network allows data consumers to interpret this data visually using tools including dynamic timeline maps, infographics, advanced charting and a streamlined user interface designed to be simple to use. With varying user levels in mind, this collection of tools provides a variety of avenues for the users to explore the data. Visualization results can be exported and embedded into other websites with data interpretation statements, benchmarks, and other visual cues that allow a broad audience to be able to access to environmental public health surveillance data.

\section{Conclusions}

While the internet contains a wealth of health and environmental datasets, the Tracking Network provides a centralized location to access over 450 environmental health measures and provides a variety of visualization tools to translate the data into useful information. The speaker will present a range of display options available on the Tracking Network, highlight ways to present data for easy understanding and consumption, and provide a brief look into the future of data visualization. 